\title{
Caracterização físico-química, reológica e sensorial de queijos tipo Prato com teor reduzido de gordura
}

\author{
Chemical composition, rheological and sensorial characterization of reduced fat Prato cheese
}

\section{Christiane Maciel Vasconcellos Barros DE RENSIS ${ }^{1 \star}$, Ademir José PETENATE ${ }^{2}$, Walkiria Hanada VIOTTO}

\section{Resumo}

O objetivo deste trabalho foi caracterizar 3 marcas diferentes de queijos tipo Prato com teor reduzido de gordura, comercializados em Campinas - SP, quanto a sua composição, proteólise, capacidade de derretimento, propriedades reológicas e aceitação sensorial. Não houve diferença significativa $(\mathrm{p}>0,05)$ na composição dos queijos. A extensão e a profundidade de proteólise foram significativamente maiores $(\mathrm{p}<0,05)$ ao final de 60 dias de armazenamento refrigerado para o queijo da marca B. Os queijos das marcas A e C se apresentaram menos macios e mais elásticos ao final de 60 dias de armazenamento refrigerado. Nos testes de aceitação sensorial, o queijo da marca B obteve notas significativamente mais altas $(\mathrm{p}<0,05)$ para os atributos sabor e impressão global, sendo que $70 \%$ dos consumidores certamente ou provavelmente comprariam esse queijo.

Palavras-chave: queijo tipo Prato; teor reduzido de gordura; proteólise; reologia; sensorial.

\begin{abstract}
Composition, proteolysis, melting, reological properties, and sensory acceptability of three commercial reduced fat Prato cheese, found in the market of Campinas - SP, Brazil, were evaluated. There was no statistical difference $(p>0,05)$ in the cheese composition. The proteolysis extension and depth were significantly higher $(\mathrm{p}<0.05)$ at 60 days of refrigerated store for cheese B. Cheeses A and C were firmer and more elastic at 60 days of refrigerated store. Sensory acceptability tests indicated significant differences $(\mathrm{p}<0.05)$ among the cheeses for flavor and global impression. Cheese B was better graded than the other cheeses in the sensory acceptance test and its buying intention scores indicated that $70 \%$ consumers would certainly or probably buy this cheese.
\end{abstract}

Keywords: Prato cheese; reduced fat; proteolysis; rheology; sensory acceptability.

\section{Introdução}

O queijo tipo Prato, um queijo de massa semicozida e lavada, se caracteriza pelo seu sabor suave e consistência macia. Atualmente, é o segundo queijo mais consumido no Brasil, principalmente na forma de sanduíches ou como ingrediente culinário (COSTA JÚNIOR; PINHEIRO, 1998).

Esse tipo de queijo, assim como a maioria dos queijos produzidos no País, é fabricado a partir de leite integral. Nas últimas décadas, porém, a crescente preocupação do consumidor em relação à saúde tem causado aumento no consumo de alimentos de baixo teor de gordura $(\mathrm{AKOH}, 1998$; KONDYLI et al., 2002), o que tem levado a indústria láctea a produzir queijo tipo Prato com teor reduzido de gordura.

É desejável que um queijo tipo Prato com teor reduzido de gordura seja similar em sabor, firmeza e textura ao queijo integral. A textura dos queijos é afetada por mudanças na composição, microestrutura, estado físico-químico dos componentes do queijo, força das interações entre os elementos estruturais que o compõem e de sua macroestrutura (DRAKE; BOYLSTON; SWANSON, 1996b).
Em geral, queijos com teor reduzido de gordura são menos aceitos pelos consumidores por apresentarem textura mais dura, ausência de aroma característico e problemas de sabor (amargo e de baixa intensidade) (CUNHA, 2002; DRAKE; BOYLSTON; SWANSON, 1996a; JONHSON; CHEN, 1995).

Várias alternativas têm sido usadas pelas indústrias de laticínios para melhorar a qualidade sensorial dos queijos com baixo teor de gordura, tais como: a) modificações na tecnologia de fabricação; b) uso de culturas adjuntas; e c) o uso de miméticos.

Não há na literatura nenhum trabalho sobre as características físico-químicas e sensoriais do queijo tipo Prato com teor reduzido de gordura. O objetivo desse trabalho foi caracterizar os queijos tipo Prato com teor reduzido de gordura comercializados em Campinas - SP, quanto a sua composição, proteólise, propriedades reológicas, microestrutura e aceitação sensorial.

${ }^{1}$ Ciência e Tecnologia do Leite - UNOPAR, Av. Paris, 675, Jd. Piza. CEP 86041-120, Londrina - PR, Brasil, E-mail: christiane@unopar.br

${ }^{2}$ Instituto de Matemática, Estatística e Computação Científica, Universidade Estadual de Campinas - UNICAMP

${ }^{3}$ Engenharia de Alimentos, Universidade Estadual de Campinas - UNICAMP

${ }^{*}$ A quem a correspondência deve ser enviada 


\section{Material e métodos}

\subsection{Amostragem}

Amostras comerciais de três marcas diferentes de queijos tipo Prato com teor reduzido de gordura foram adquiridas em supermercados da cidade de Campinas, Estado de São Paulo. Duas marcas eram provenientes de laticínios do Estado de São Paulo e a terceira de Minas Gerais. As marcas foram selecionadas de maneira que apresentassem datas de fabricação semelhantes (entre 30-40 dias).

Oito peças ( $500 \mathrm{~g} /$ do mesmo lote), de cada marca de queijo, foram adquiridas e numeradas de um a oito para serem sorteadas em cada dia de análise.

Para todas as análises, foram retiradas e desprezadas, fatias de aproximadamente $0,5 \mathrm{~cm}$ das laterais e das partes superior e inferior. A parte central foi preparada em função de cada tipo de análise. Para a composição química, pH e acidez titulável, a parte central da peça foi cortada em cubos e triturada em multiprocessador, até obtenção de partículas de 2-3 mm. O material assim obtido foi homogeneizado manualmente, acondicionado em frascos de vidro que foram mantidos sob refrigeração $\left(12^{\circ} \mathrm{C}\right)$ até o momento das análises. Para a determinação da capacidade de derretimento, análises reológicas, e análise sensorial, foram adotados preparos diferenciados das amostras, mostrados nos itens Capacidade de derretimento, 2.4 e 2.5 , respectivamente.

As três marcas selecionadas apresentavam concentrado proteico de soro (CPS) em sua formulação e uma delas apresentava, também, a adição de cultura adjunta.

\subsection{Composição química}

Os queijos, ao serem adquiridos, foram submetidos, a análises de: $\mathrm{pH}$, pelo método potenciométrico; acidez titulável (AOAC, 1995); gordura, pelo método de Gerber (KOSIKOWSKI; MISTRY, 1997); umidade, pelo método gravimétrico, em estufa de circulação forçada, a $100^{\circ} \mathrm{C} / 24$ horas (AOAC, 1995); sal, pelo método de Volhard (RICHARDSON, 1985); cálcio, por digestão úmida do queijo, seguida de titulação com EDTA, na presença de murexida (TARAS, 1971); nitrogênio total, pelo método do macro Kjeldahl (AOAC, 1995); nitrogênio não proteico, através da determinação de nitrogênio total na fração solúvel em ácido tricloroacético (TCA) 12\% (AOAC, 1995); e nitrogênio não caseico, através da determinação de nitrogênio total na fração solúvel a pH 4,6 (BYNUM; BARBANO, 1985). Os valores de nitrogênio foram multiplicados pelo fator 6,38 para obtenção dos valores equivalentes de proteína. Todas as análises foram realizadas em triplicatas exceto $\mathrm{pH}$ e acidez titulável (duplicata).

\subsection{Mudanças ocorridas durante o armazenamento refrigerado}

\section{Avaliação do pH e da acidez titulável}

Após 30 e 60 dias de armazenamento refrigerado a $12{ }^{\circ} \mathrm{C}$, $\mathrm{o} \mathrm{pH}$ e a acidez titulável do queijo foram avaliados. $\mathrm{O} \mathrm{pH}$ foi determinado pelo método potenciométrico e a acidez titulável foi realizada conforme AOAC (1995). As duas análises foram realizadas em duplicata.

\section{Nitrogênio solúvel a pH 4,6 e em TCA 12\%}

A proteólise dos queijos foi monitorada após 30 e 60 dias de armazenamento refrigerado $\left(12^{\circ} \mathrm{C}\right)$ através da determinação dos teores de nitrogênio solúvel em tampão acetato a $\mathrm{pH}$ 4,6 e em ácido tricloroacético $12 \%$. As análises foram realizadas em triplicata, de acordo com Bynum e Barbano (1985), seguido pelo método do macro Kjeldahl (AOAC, 1995).

Os índices de extensão e profundidade de proteólise foram determinados de acordo com as Equações 1 e 2, respectivamente:

\% Extensão da proteólise $=(\%$ nitrogênio solúvel a pH 4,6 /

$\%$ nitrogênio total) $\times 100$

$\%$ Profundidade da proteólise $=(\%$ nitrogênio solúvel em TCA 12\% $/$

$\%$ nitrogênio total) $\times 100$

\section{Capacidade de derretimento}

Após 30 e 60 dias de armazenamento refrigerado a $12{ }^{\circ} \mathrm{C}$, os queijos foram avaliados quanto à capacidade de derretimento. Um cilindro da parte central da peça foi retirado com o auxílio de uma sonda de aço inox de $36 \mathrm{~mm}$ de diâmetro interno. $\mathrm{O}$ cilindro foi fracionado transversalmente com um fatiador de ovo modificado para obter cilindros de $6 \mathrm{~mm}$ de altura. $O$ teste foi realizado em seis replicatas segundo o método modificado de Schreiber descrito por Yun et al. (1993).

\subsection{Propriedades reológicas dos queijos: teste de relaxação}

O comportamento reológico dos queijos foi avaliado após 30 e 60 dias de armazenamento refrigerado, através de relaxação. As análises foram realizadas em um texturômetro TA-XT2I, da Texas Instrumentos, ajustado com uma célula de carga de $50 \mathrm{~kg}$. Para o preparo das amostras foram retirados 6 cilindros de $24 \mathrm{~mm}$ de altura por $20 \mathrm{~mm}$ de diâmetro de cada queijo, não sendo utilizados o centro e as bordas. Os cilindros foram então embalados individualmente em filme PVC, acondicionados em sacos plásticos resistentes à penetração de líquidos e mantidos em banho de água gelada a $10{ }^{\circ} \mathrm{C}$ por pelo menos 1 hora e 30 minutos antes do início dos testes. As amostras foram comprimidas por uma placa de alumínio de $35 \mathrm{~mm}$ de diâmetro.

Os testes de relaxação foram realizados conforme Cunha (2002).

\subsection{Análise sensorial}

Os queijos tipo Prato com teor reduzido de gordura foram submetidos a um teste sensorial de aceitação aos 60 dias de armazenamento refrigerado. Foram recrutados 40 provadores não treinados dentre alunos e funcionários da UNICAMP. Os provadores foram selecionados em função de consumirem queijo na versão integral e/ou na versão light, disponibilidade e 
interesse em participar do teste. Os testes foram realizados em cabines individuais do Laboratório de Análise Sensorial do DTA/ FEA/Unicamp, sob luz branca. As amostras codificadas com três dígitos foram apresentadas de forma monádica em pratos descartáveis brancos, cortadas em pedaços de aproximadamente $3 \mathrm{~cm} \times 2 \mathrm{~cm} \times 0,5 \mathrm{~cm}$. A temperatura de apresentação foi de $12^{\circ} \mathrm{C}$. Água e biscoito água e sal foram servidos para o consumo entre a avaliação das amostras. A ordem de apresentação das amostras foi aleatória.

Os atributos aparência, aroma, sabor, textura e impressão global foram analisados com a utilização de escala estruturada de nove pontos, na qual $1=$ desgostei muito e $9=$ gostei muito. Para a intenção de compra foi utilizada escala estruturada de cinco pontos, na qual $1=$ certamente não compraria $\mathrm{e}$ $5=$ certamente compraria.

\subsection{Planejamento experimental e análise estatística dos resultados}

Para a avaliação da composição química foi realizado um experimento aleatorizado, com três blocos, sendo a data de fabricação o fator utilizado para a montagem dos blocos. Em cada bloco foram avaliados três marcas comerciais de queijo tipo Prato com teor reduzido de gordura. Os resultados da composição química foram analisados através de Análise de Variância (ANOVA), utilizando-se o teste de Duncan (5\% de probabilidade) para verificar diferenças entre as amostras.

Para a avaliação do $\mathrm{pH}$, acidez titulável, proteólise, capacidade de derretimento e propriedades viscoelásticas foi realizado um plano experimental split-plot, sendo a subparcela obtida pela incorporação do fator tempo de armazenamento refrigerado (t). As análises foram realizadas nos dias 30 e 60 de armazenamento refrigerado. O teste de F-ANOVA foi usado para avaliar as diferenças entre as marcas de queijo, entre tempos e a interação tempo versus marcas. Foi utilizado o teste de Duncan de comparações múltiplas para agrupar marcas e/ou tempos com médias cujas diferenças não foram estatisticamente significativas.

Os resultados obtidos na análise sensorial foram analisados através de Análise de Variância (ANOVA) e do teste de Duncan (5\% de probabilidade).

\section{Resultados e discussão}

\subsection{Composição química dos queijos}

A Tabela 1 apresenta a composição química média das três marcas dos queijos tipo Prato com teor reduzido de gordura adquiridos no mercado de Campinas - SP.

Os dados apresentados na Tabela 1 demonstram que não houve diferença significativa em relação à composição química das marcas analisadas ao nível de $5 \%$ de significância.

Considerando que o teor de gordura de um queijo tipo Prato integral é em média 28\% (FURTADO; LOURENÇO NETO, 1994), as reduções observadas nos queijos A, B e C foram, respectivamente, de 33, 26,4 e 34\% de gordura. Logo, todas as marcas apresentaram a redução mínima no teor de gordura (25\%) exigida pela legislação brasileira para produtos com teor reduzido de gordura (BRASIL, 1998).

Verificou-se também que todos os queijos apresentaram teor de umidade superior ao do queijo tipo Prato integral ( 43\%). Esse aumento está relacionado com a diminuição do teor de gordura dos queijos, que promove uma alteração no balanço dos componentes.

\subsection{Mudanças ocorridas durante o armazenamento refrigerado}

\section{Evolução do pH e acidez titulável}

Observa-se na Tabela 2, que os queijos apresentaram diferenças não significativas para os valores de $\mathrm{pH}$ e acidez titulável, em relação às marcas $(\mathrm{p}=0,492 \mathrm{ep}=0,892)$ e durante $\mathrm{o}$ tempo de armazenamento refrigerado $(\mathrm{p}=0,649 \mathrm{e} \mathrm{p}=0,112)$.

\section{Evolução da proteólise}

A Tabela 2 mostra os resultados da análise estatística dos índices de extensão e profundidade da proteólise dos queijos tipo Prato com teor reduzido de gordura ao longo do tempo de armazenamento.

A extensão da proteólise é um fator indicativo da proteólise primária e ocorre principalmente pela ação proteolítica do coalho ou coagulante residual no queijo, sobre a $\alpha_{\mathrm{s} 1}$-caseína e, em menor escala, sobre a $\beta$-caseína, dando origem a peptídeos de alto e médio peso molecular (CHOISY et al., 1987; FOX, 1989).

O tipo de marca influenciou significativamente $(p=0,0045)$ a extensão da proteólise, sendo que a marca $B$ foi a que apresentou maior índice. Provavelmente, nessa marca pode ter sido adicionado uma quantidade maior de coalho do que nas outras duas, tendo em vista que o coalho é o principal responsável pela proteólise primária.

Para todas as marcas, houve um aumento significativo nos índices de extensão da proteólise com o tempo $(\mathrm{p}=0,0032)$, como mostra a Figura 1.

Os resultados alcançados no presente estudo demonstram que houve influência quanto à marca comercial investigada na extensão da proteólise.

Em relação à profundidade da proteólise, índice este relacionado à presença de enzimas proteolíticas oriundas de microrganismos que hidrolisam os peptídeos resultantes da ação do coalho e da plasmina sobre as caseínas (FOX, 1989), os resultados deste estudo mostraram diferenças significativas nos índices encontrados na comparação das três marcas comerciais dos queijos $(\mathrm{p}=0,0321)$.

Para as marcas A e B, ocorreu um aumento significativo nos índices de profundidade (\%NS (TCA $12 \%$ )/NT) com o tempo de armazenamento $(p=0,000)$, sendo que marca $B$ foi a que apresentou os maiores índices. A Tabela 2 mostra que 
Tabela 1. Composição média $(n=3)$ de três marcas de queijos Prato com reduzido teor de gordura.

\begin{tabular}{|c|c|c|c|}
\hline Componente & Marca A & Marca B & Marca C \\
\hline Umidade (\%) & $50,73+2,63^{a}$ & $45,98+1,50^{\mathrm{a}}$ & $50,29+2,57^{a}$ \\
\hline Gordura (\%) & $18,75+2,41^{\mathrm{a}}$ & $20,60+1,51^{a}$ & $18,58+1,88^{a}$ \\
\hline Gordura em base seca $(\%)^{1}$ & $37,96+3,12^{\mathrm{a}}$ & $38,14+3,25^{\mathrm{a}}$ & $37,34+2,49^{a}$ \\
\hline Caseína (\%) & $21,97+1,73^{\mathrm{a}}$ & $21,22+1,32^{\mathrm{a}}$ & $22,18+2,29^{a}$ \\
\hline Sal (\%) & $1,61+0,58^{\mathrm{a}}$ & $1,66+0,59^{a}$ & $1,21+0,34^{\mathrm{a}}$ \\
\hline$C: G^{3}$ & $1,18+0,07^{\mathrm{a}}$ & $1,04+0,14^{\mathrm{a}}$ & $1,20+0,14^{\mathrm{a}}$ \\
\hline Acidez (\%ácido láctico) & $0,57+0,17^{\mathrm{a}}$ & $0,59+0,15^{\mathrm{a}}$ & $0,59+0,42^{a}$ \\
\hline $\mathrm{pH}$ & $5,38+0,21^{\mathrm{a}}$ & $5,52+0,12^{\mathrm{a}}$ & $5,42+0,31^{\mathrm{a}}$ \\
\hline
\end{tabular}

${ }^{1}$ Gordura em base seca, ${ }^{2}$ relação sal/umidade, ${ }^{3}$ relação caseína/gordura; em uma mesma linha, médias acompanhadas de letras em comum, não diferem entre si pelo teste de Duncan $(\mathrm{p}>0,05)$.

Tabela 2. Quadrados médios e probabilidades para evolução do $\mathrm{pH}$, da acidez titulável, da extensão da proteólise e da profundidade da proteólise dos queijos Prato comerciais com reduzido teor de gordura durante o armazenamento refrigerado a $12{ }^{\circ} \mathrm{C}$.

\begin{tabular}{|c|c|c|c|c|c|c|c|c|c|}
\hline \multirow[t]{3}{*}{ Fatores } & \multirow[b]{3}{*}{ GL } & \multirow{2}{*}{\multicolumn{2}{|c|}{$\mathrm{pH}$}} & \multirow{2}{*}{\multicolumn{2}{|c|}{ Acidez titulável }} & \multicolumn{4}{|c|}{ Nitrogênio solúvel } \\
\hline & & & & & & \multicolumn{2}{|c|}{$\mathrm{pH} \mathrm{4,6}$} & \multicolumn{2}{|c|}{ TCA $12 \%$} \\
\hline & & QM & $\mathrm{P}$ & $\mathrm{QM}$ & $\mathrm{P}$ & $\mathrm{QM}$ & $\mathrm{P}$ & $\mathrm{QM}$ & $\mathrm{P}$ \\
\hline $\operatorname{Marcas}(\mathrm{M})$ & 2 & 0,0954 & 0,492 & 0,2300 & 0,892 & 95,5584 & $0,0045^{\star}$ & 53,8883 & $0,0321^{\star}$ \\
\hline Erro(a) & 4 & 0,1121 & - & 0,1951 & - & 3,4498 & - & 5,8781 & - \\
\hline Tempo $(\mathrm{t})$ & 1 & 0,0054 & 0,649 & 0,0378 & 0,112 & 49,2440 & $0,0032^{\star}$ & 36,2542 & $0,0005^{\star}$ \\
\hline Interação $\left(M^{\star} t\right)$ & 2 & 0,0031 & 0,897 & 0,0072 & 0,551 & 0,1457 & 0,9598 & 5,2173 & $0,0314^{\star}$ \\
\hline Erro(b) & 6 & 0,2333 & - & 0,0109 & - & 3,5267 & - & 0,8010 & - \\
\hline
\end{tabular}

$\mathrm{GL}=$ graus de liberdade; $\mathrm{QM}=$ quadrado médio; $\mathrm{e}$ = probabilidade.

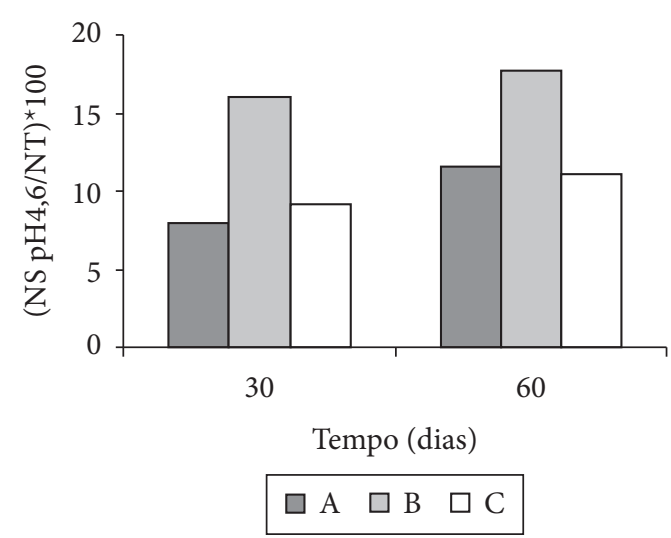

Figura 1. Evolução da extensão da proteólise nos queijos tipo Prato comerciais com teor reduzido de gordura durante o armazenamento refrigerado a $12{ }^{\circ} \mathrm{C}$.

a interação entre a marca e o tempo também foi significativa $(\mathrm{p}=0,0314)$.

A Figura 2 mostra que o queijo B se comportou de maneira diferente dos outros queijos ao longo do tempo. Essa diferença provavelmente está relacionada à adição de uma cultura adjunta (constituída de Streptococcus thermophilus) juntamente com o fermento mesófilo ao leite de fabricação do queijo da marca B (Dados fornecidos pelo fabricante).

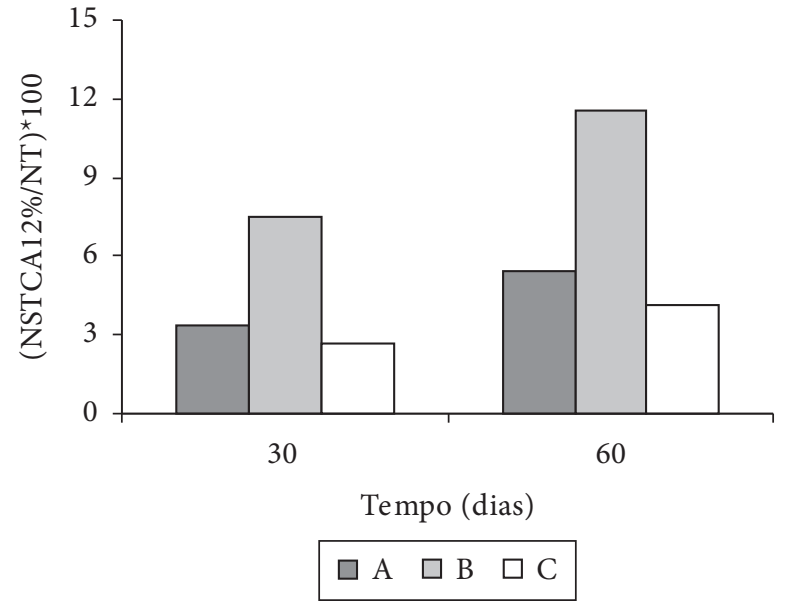

Figura 2. Evolução da profundidade da proteólise nos queijos tipo Prato comerciais com teor reduzido de gordura durante o armazenamento refrigerado a $12^{\circ} \mathrm{C}$.

As culturas adjuntas são adicionadas intencionalmente ao leite destinado à fabricação de queijos, para melhorar ou acelerar o desenvolvimento de sabor dos queijos com teor reduzido de gordura através do aumento da proteólise, especificamente atividade aminopeptidase, a qual reduz o sabor amargo e aumenta a concentração de peptídeos de sabor desejável e precursores voláteis de sabor (MISTRY, 2001; FOX et al., 1998; DRAKE; BOYLSTON; SWANSON, 1996a). 


\section{Capacidade de derretimento}

A capacidade de derretimento é uma característica importante para queijos, como o caso do tipo Prato utilizado como ingrediente culinário.

De acordo com os resultados encontrados, verificou-se que a marca do queijo não afetou significativamente $(\mathrm{p}=1,6733)$ a capacidade de derretimento dos queijos. Entretanto, observouse um aumento da capacidade de derretimento $(\mathrm{p}=0,0037)$ ao longo do tempo para as três marcas de queijo (Figura 3). Esse aumento está diretamente ligado ao aumento do índice de extensão da proteólise durante o armazenamento. A proteólise primária, cujo principal responsável é o coalho ou coagulante residual, está relacionada com o aumento da capacidade de derretimento do queijo devido ao enfraquecimento da matriz proteica que faz com que o queijo perca a capacidade de manter sua estrutura durante o aquecimento (KINDSTEDT, 1993).

Estudo conduzido por Spadoti (2003) demonstrou que a capacidade de derretimento de queijo tipo Prato integral é mais elevada. Esse resultado pode ser explicado considerandose que a remoção da gordura aumenta proporcionalmente o teor de proteína e assim a matriz proteica se torna mais rígida e mais compacta, com menor tendência a derreter e fluir (MISTRY, 2001).

\subsection{Propriedades reológicas dos queijos: teste de relaxação}

O modelo generalizado de Maxwell utilizado neste estudo foi capaz de descrever satisfatoriamente o comportamento viscoelástico dos queijos, já que as curvas experimentais obtidas apresentaram boa correlação $\left(\mathrm{R}^{2}>0,99\right)$. As curvas experimentais foram ajustadas utilizando-se o programa Statistica ${ }^{\oplus}$. Para o cálculo dos módulos de elasticidade $\mathrm{E}_{\mathrm{o}}, \mathrm{E}_{1}$, $E_{2}$ e das viscosidades $\eta_{1}$ e $\eta_{2}$, foram utilizados os parâmetros $\mathrm{S}_{\mathrm{o}}, \mathrm{S}_{1}, \mathrm{~S}_{2}, \zeta_{1}$ e $\zeta_{2}$, respectivamente, conforme descrito por Cunha (2002).

$\mathrm{Na}$ análise dos resultados, verifica-se novamente que a marca influenciou significativamente $(\mathrm{p}<0,05)$ quase todos os parâmetros viscoelásticos dos queijos.

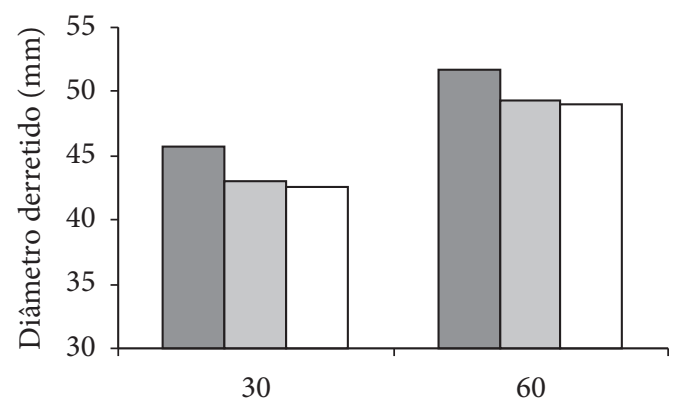

Tempo (dias)

$\square \mathrm{A} \square \mathrm{B} \quad \square \mathrm{C}$

Figura 3. Evolução da capacidade de derretimento dos queijos tipo Prato comerciais com teor reduzido de gordura durante o armazenamento refrigerado a $12^{\circ} \mathrm{C}$.
O teste de Duncan de comparação múltipla revelou que as marcas B e C diferiram significativamente $(\mathrm{p}<0,05)$ da marca A. Os valores dos módulos de elasticidade e viscosidade da marca A foram superiores aos valores das marcas B e C.

A elasticidade é definida como a tendência do material de recuperar sua forma original quando uma tensão aplicada é removida. Isso significa que quanto maior a tendência à recuperação maior será a elasticidade. Já a viscosidade é uma medida da resistência do corpo à deformação, quando este é submetido a uma tensão e reflete na maciez do queijo. Quanto maior a resistência à deformação, menor será a maciez do queijo (FOX, 1998). Pode-se dizer, então, que a marca A apresentou-se menos macia e mais elástica que as marcas B e C.

A Tabela 3 mostra que, durante o tempo de armazenamento dos queijos (30-60 dias), a variação dos parâmetros também foi significativa $(\mathrm{p}<0,05)$. Os módulos de elasticidade e a viscosidade de todos os elementos do modelo diminuíram durante o armazenamento refrigerado para essas duas marcas. A redução do módulo de elasticidade indica uma diminuição na tendência do material a recuperar sua forma original, quando uma tensão aplicada é removida. Essa diminuição pode ser relacionada com a proteólise primária que provocou uma redução na firmeza e na elasticidade dos queijos, tornandoos mais macios, menos firmes e menos elásticos. Durante a proteólise secundária, enzimas provenientes da cultura láctica, cultura adjunta ou de bactérias contaminantes podem ser responsáveis pela continuação do processo (FOX, 1989). Ainda, em relação à viscosidade, nota-se que sua diminuição em função do tempo implica em as marcas B e C apresentarem uma textura mais macia e menos firme no final do tempo de armazenamento refrigerado. Esses resultados concordam com os resultados obtidos para os índices de extensão e profundidade (Tabela 2) durante o armazenamento refrigerado.

Finalmente, a interação entre a marca e o tempo também foi significativa para todos os parâmetros do modelo, indicando que as marcas se comportaram de maneira diferente ao longo do tempo.

\subsection{Análise sensorial}

As médias de aceitação dos queijos tipo Prato comerciais com teor reduzido de gordura avaliados após 60 dias de armazenamento refrigerado encontram-se expressas na Tabela 4, para os atributos aparência, aroma, sabor, textura e impressão global. Em geral, as amostras apresentaram boa aceitação, com médias variando entre 6 (gostei ligeiramente) e 7 (gostei moderadamente) para sabor e impressão global. Os atributos de aparência, aroma e textura obtiveram notas mais elevadas.

Os resultados apresentados na Tabela 4 demonstram que não houve diferença significativa $(\mathrm{p}<0,05)$ na aceitação dos queijos para os atributos aparência, aroma, textura e impressão global.

Apesar do teor reduzido de gordura dos queijos comerciais, as médias para o atributo textura foram altas. Tal fato pode ser explicado pela adição de concentrado proteico de soro 
Tabela 3. Valores dos módulos de elasticidade $E_{0}, E_{1}, E_{2}$ e das viscosidades $\eta_{1}$ e $\eta_{2}$ dos queijos tipo Prato comerciais com teor reduzido de gordura durante o armazenamento refrigerado a $12^{\circ} \mathrm{C}$.

\begin{tabular}{|c|c|c|c|c|c|c|c|c|c|c|}
\hline & \multicolumn{2}{|c|}{$\mathrm{E}_{\mathrm{o}}$} & \multicolumn{2}{|c|}{$\mathrm{E}_{1}$} & \multicolumn{2}{|c|}{$\mathrm{E}_{2}$} & \multicolumn{2}{|c|}{$\eta_{1}$} & \multicolumn{2}{|c|}{$\eta_{2}$} \\
\hline & Dia 30 & Dia 60 & Dia 30 & Dia 60 & Dia 30 & Dia 60 & Dia 30 & Dia 60 & Dia 30 & Dia 60 \\
\hline Marca A & $26254,24^{\mathrm{a}}$ & $22902,22^{b}$ & $87541,13^{a}$ & $69711,15^{\mathrm{b}}$ & $71050,36^{a}$ & $55879,31^{\mathrm{b}}$ & $964389,75^{\mathrm{a}}$ & $729279,63^{b}$ & $13580756,42^{a}$ & $10268863,8^{b}$ \\
\hline Marca B & $18496,10^{\mathrm{a}}$ & $92850,97^{\mathrm{b}}$ & $95535,63^{a}$ & $44108,87^{b}$ & $73257,31^{\mathrm{a}}$ & $34125,41^{b}$ & $901302,75^{\mathrm{a}}$ & $457204,46^{\mathrm{b}}$ & $10665615,95^{a}$ & $4908300,56^{\mathrm{b}}$ \\
\hline Marca C & $20960,41^{a}$ & $17312,44^{\mathrm{b}}$ & $77679,05^{\mathrm{a}}$ & $47423,82^{\mathrm{b}}$ & $56785,40^{\mathrm{a}}$ & $39298,77^{\mathrm{b}}$ & $756261,93^{\mathrm{a}}$ & $479589,56^{\mathrm{b}}$ & $10259382,25^{\mathrm{a}}$ & $6917495,00^{\mathrm{b}}$ \\
\hline
\end{tabular}

Para cada parâmetro, em uma mesma linha, médias acompanhadas de letras em comum, não diferem entre si pelo teste de Duncan ( $\mathrm{p}>0,05)$.

Tabela 4. Médias de aceitação atribuídas pelos provadores para as amostras de queijos Prato comerciais de reduzido teor de gordura. ( $\mathrm{n}=40$ provadores).

\begin{tabular}{lccc}
\hline \multicolumn{1}{c}{ Atributos } & $\mathrm{A}$ & $\mathrm{B}$ & $\mathrm{C}$ \\
\hline Aparência $^{1}$ & $7,35^{\mathrm{a}}$ & $7,48^{\mathrm{a}}$ & $7,63^{\mathrm{a}}$ \\
Aroma & $7,38^{\mathrm{a}}$ & $7,48^{\mathrm{a}}$ & $7,35^{\mathrm{a}}$ \\
Sabor & $6,38^{\mathrm{b}}$ & $7,23^{\mathrm{a}}$ & $6,30^{\mathrm{b}}$ \\
Textura & $7,23^{\mathrm{a}}$ & $7,58^{\mathrm{a}}$ & $7,30^{\mathrm{a}}$ \\
Impressão global & $6,90^{\mathrm{a}}$ & $7,28^{\mathrm{a}}$ & $6,98^{\mathrm{a}}$ \\
Intenção de compra $^{2}$ & $3,70^{\mathrm{b}}$ & $4,13^{\mathrm{a}}$ & $3,65^{\mathrm{b}}$ \\
\hline
\end{tabular}

Em uma mesma linha, médias acompanhadas de letras em comum, não diferem entre si pelo este de Duncan $(\mathrm{p}>0,05) ;{ }^{1} 1=$ desgostei muito, $9=$ gostei muito; $\mathrm{e}^{2} 1=$ certamente não compraria, 5 = certamente compraria.

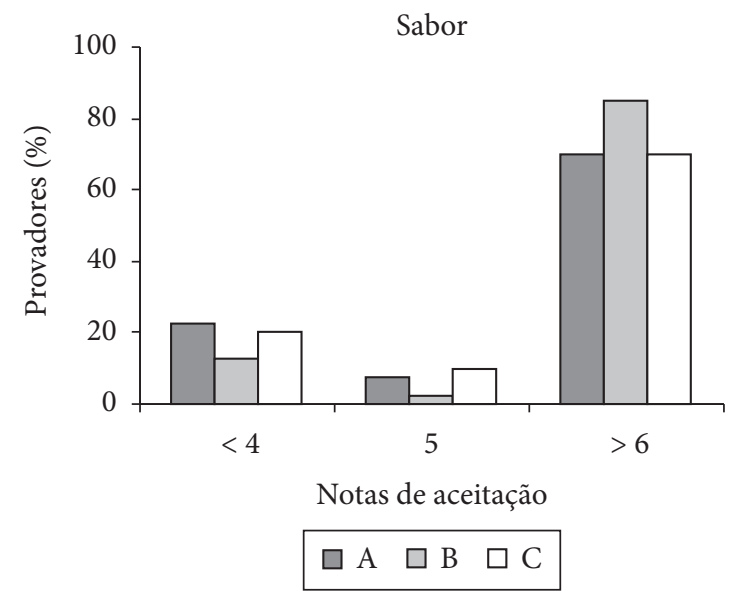

Figura 4. Histograma de frequências das notas de aceitação dos queijos tipo Prato comerciais com teor reduzido de gordura em relação ao atributo sabor.

(CPS) usado como mimetizador de gordura, o que aumenta a capacidade de retenção de água. Nesse caso, a água substituiu em certo grau o papel da gordura, atuando como lubrificante entre os agregados de caseína, fazendo com que o queijo apresentasse uma textura mais macia, que provavelmente agradou aos provadores.

O queijo B apresentou médias de aceitação para os atributos sabor (Figura 4) e intenção de compra significativamente $(\mathrm{p}<0,05)$ superiores às demais amostras. O melhor sabor possivelmente deveu-se à ação da cultura adjunta adicionada a este queijo, a qual, no decorrer do tempo de armazenamento

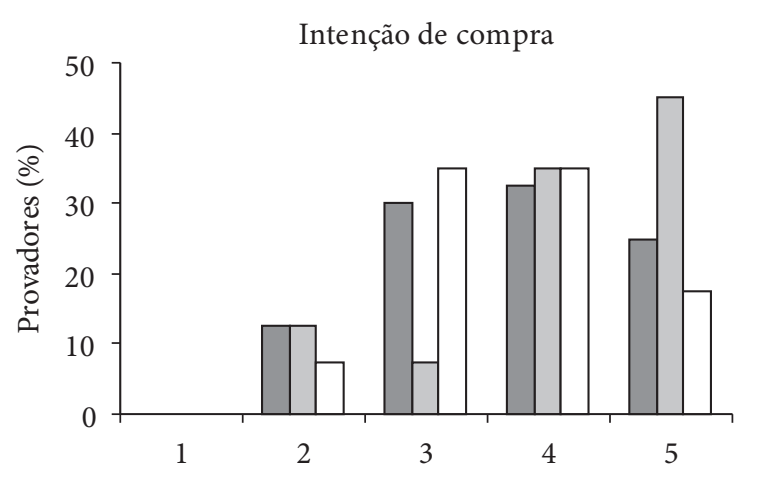

1 = certamente não compraria $/ 5$ = certamente compraria

$\square$ A $\square$ B $\square \mathrm{C}$

Figura 5. Histograma de frequências da avaliação sensorial dos queijos tipo Prato comerciais com teor reduzido de gordura em relação à intenção de compra.

refrigerado aumentou os índices de proteólise secundária através do aumento da atividade proteolítica.

A Figura 5 mostra o histograma de frequências para o atributo intenção de compra. A percepção de um melhor sabor na marca B foi decisiva na intenção de compra do queijo pelos consumidores. Cerca de $70 \%$ dos provadores certamente ou provavelmente comprariam o queijo tipo Prato com teor reduzido de gordura da marca $\mathrm{B}$, indicando uma melhor aceitação em relação às marcas $\mathrm{A}$ e $\mathrm{C}$.

\section{Conclusões}

Dentre as três marcas comerciais de queijo do tipo Prato investigadas, os queijos da marca $\mathrm{B}$ apresentaram os maiores índices de profundidade de proteólise, maior maciez e menor elasticidade e uma melhor aceitação sensorial. A adição de uma cultura adjunta nessa marca, provavelmente, contribuiu para a proteólise secundária mais pronunciada e, consequentemente, para melhoria nas propriedades reológicas e sensoriais dos queijos, já que $70 \%$ dos consumidores afirmaram que certamente ou provavelmente comprariam esse produto.

Essas diferenças encontradas não foram acompanhadas pela alteração na composição química dos produtos, ou seja, os valores encontrados para as três marcas não apresentaram diferenças significativas entre si. 


\section{Referências bibliográficas}

AKOH, C. C. Fat replacers. Food Technology, v. 52, n. 3, p. 47-53, 1998.

Association of Official Analytical Chemistry - AOAC. Official methods of analysis. $15 \mathrm{ed}$. Washington, 1995.

BYNUM, D. G.; BARBANO, D. M. Whole milk reverse osmosis retentates for cheddar cheese manufacturing: chemical changes during ageing. Journal of Dairy Science, v. 68, n. 1, p. 1-10, 1985.

CHOISY, C. et al. Les phénomènes microbiologiques et enzymatiques et la biochimie de l'affinage. In: ECK, A. (Ed.). Le fromage. Paris: Techinique et Documentation, 1987. p. 62-100.

COSTA JÚNIOR, L. C. G.; PINHEIRO, A. J. R. Influência da relação caseína/gordura nas características físico-químicas do queijo prato. Revista do Instituto de Laticínios Cândido Tostes, v. 305, n. 53, p. 29-49, 1998.

CUNHA, C. R. Efeito do uso de retentados de baixo fator de concentração no rendimento, proteólise e propriedades viscoelásticas de queijo minas frescal de baixo teor de gordura fabricado a partir de leite ultrafiltrado. Campinas, 2002. $114 \mathrm{p}$. Dissertação (Mestrado em Engenharia de Alimentos) - Universidade Estadual de Campinas - UNICAMP.

DRAKE, M. A.; BOYLSTON, T. D.; SWANSON, B. G. Chemical and sensory effects of lactobacillus adjunct in cheddar cheese. Food Research International, v. 29, n. 3-4, p. 381-387, 1996a.

DRAKE, M. A.; BOYLSTON, T. D.; SWANSON, B. G. Fat mimetics in low-fat cheddar cheese. Journal of Food Science, v. 61, n. 6, p. 366-369, 1996b.

FOX, P. F. et al. Fundamentals of cheese science. Gaithersburg: Aspen, 1998. p. 587.

FOX, P. F. Proteolysis During Cheese Manufacture and Ripening. Journal of Dairy Science, v. 72, n. 6, p. 1379-1400, 1989.

FURTADO, M. M.; LOURENÇO NETO, J. P. M. L.Tecnologia de Queijos: manual técnico para a produção industrial de queijos. In: Minas Frescal. São Paulo: Dipemar, 1994. p. 73-75.
JONHSON, M. E.; CHEN, C. M. Technology of manufacturing reduced-fat cheddar cheese. In: MALIN, E. L.; TUNICK, M. H. Chemistry of structure: Function relationships in cheese. New York, London: Plenum Press, 1995. cap. 21, p. 331-338.

KINDSTEDT, P. S. Effect of manufacturing factors, composition and proteolysis on the functional characteristics of Mozzarella cheese. Critical Reviews in Food Science and Nutrition, v. 33, n. 2, p. $167-187,1993$.

KONDYLI, E. et al. Free fatty acids and volatile compounds of low-fat feta-type cheese made with a commercial adjunct culture. Food Chemistry, v. 79. n. 2, p. 199-205, 2002.

KOSIKOWSKI, F. V.; MISTRY, V. V. Cheese and fermented milk foods. 3 ed. Westport: AVI, 1997. p. 728.

BRASIL. Ministério da Saúde. Secretaria de Vigilância Sanitária. Portaria $n^{\circ} 27$, de 13 de janeiro de 1998. Regulamento técnico referente à informação nutricional complementar. Diário Oficial da União, Brasília...

MISTRY, V. V. Low fat cheese technology. International Dairy Journal, v. 11. n. 4, p. 413-422, 2001.

RICHARDSON, G. H. (Ed.). Standard methods for examination of dairy products. Washington: American Public Health Association, 1985. $345 \mathrm{p}$.

SPADOTI, L. M. Avaliação de propriedades do queijo tipo prato obtido por modificações no processo tradicional de fabricação. Campinas, 2003. 229 p. Tese (Doutorado em Tecnologia de Alimentos) - Universidade Estadual de Campinas - UNICAMP.

TARAS, M. J. Standard methods for the examination of water and wastewater. Washington: American Public Health Association, 1971. $874 \mathrm{p}$.

YUN, J. J. et al. Mozzarella cheese: impact of milling $\mathrm{pH}$ on functional properties. Journal of Dairy Science, v. 76, n. 12, p. 3639-3647, 1993. 